\title{
Bilateral tight swollen legs
}

\author{
Dorota Linda • Ashwyn Rajagopalan • Samih Salama • \\ Nader Khalidi • Srinivasan Harish
}

Published online: 22 April 2009

(C) ISS 2009

\section{History}

A 20-year-old female initially presented to the emergency room with a 3-day history of painless swelling involving both legs following exercise. A deep vein thrombosis was suspected and excluded, but she continued to have progressive symptoms in her legs over the next 8 months including pain, tightness of muscles, and skin changes (Fig. 1). Magnetic resonance imaging of the bilateral legs was performed (Fig. 2a-c) followed by full thickness skin to muscle biopsy (Fig. 3a-b).

The diagnosis can be found at http://dx.doi.org/10.1007/s00256009-0695-9.

D. Linda $\cdot$ S. Harish $(\bowtie)$

Department of Diagnostic Imaging,

St. Joseph's Healthcare and McMaster University,

Hamilton, ON, Canada L8N 2A6

e-mail: sriniharish@gmail.com
A. Rajagopalan $\cdot$ S. Salama
Department of Pathology,
St. Joseph's Healthcare and McMaster University,
Hamilton, ON, Canada
N. Khalidi
Department of Rheumatology,
St. Joseph's Healthcare and McMaster University,
Hamilton, ON, Canada

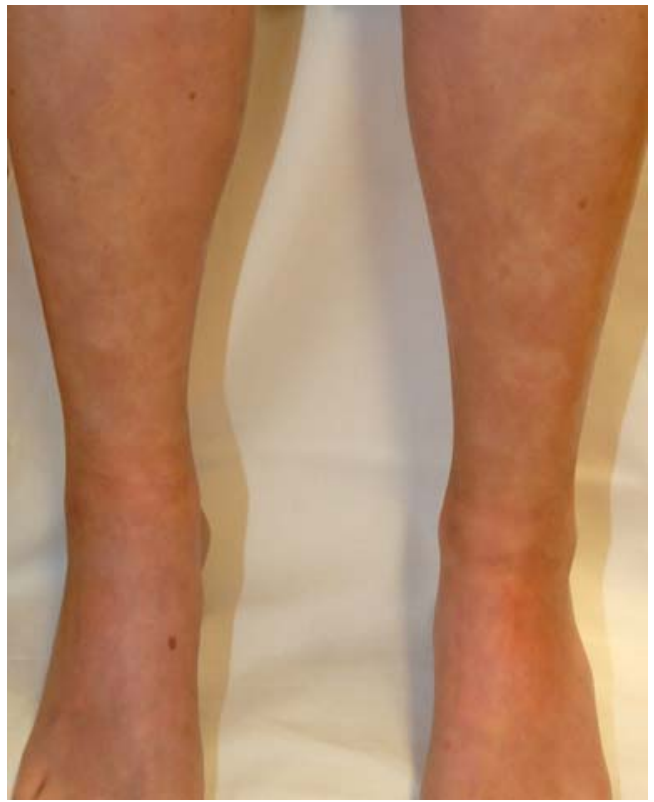

Fig. 1 Photograph of bilateral shins 

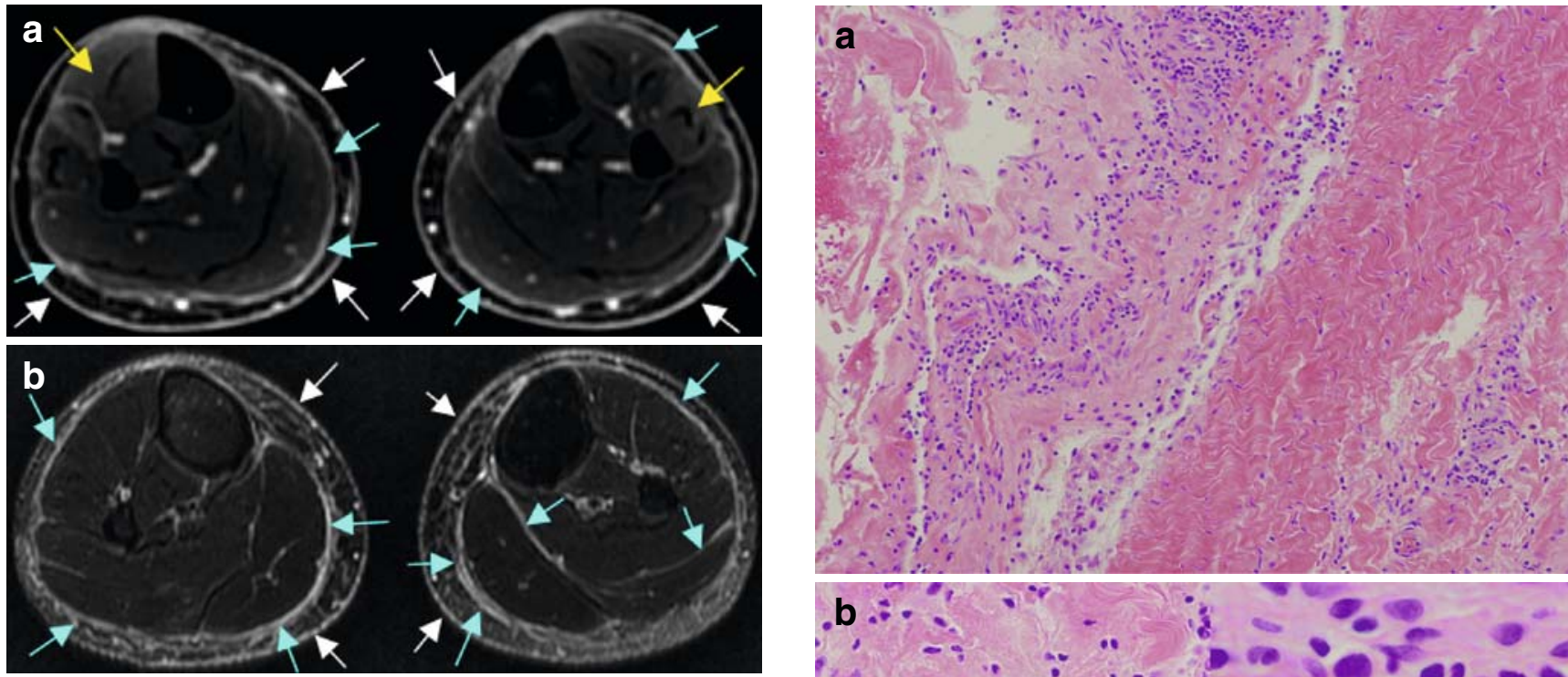

C

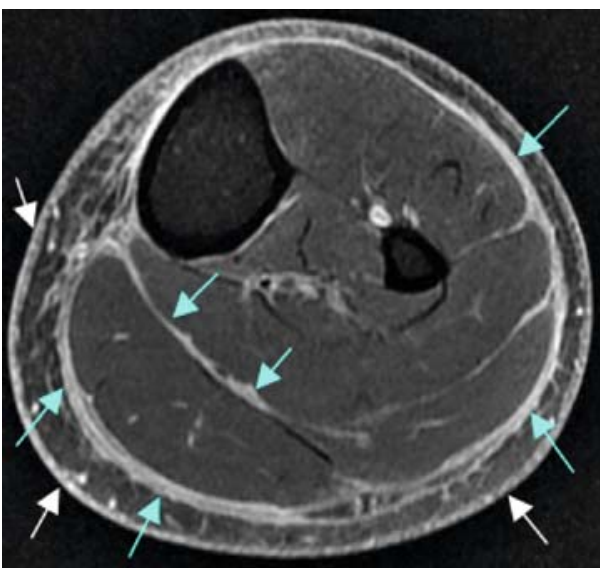

Fig. 2 a Axial post gadolinium 3D fast acquisition with multiphase EFGRE sequence through both calves (giving a T1-weighted fatsaturated type weighting). b Axial FSE T2-weighted fat-saturated sequence through both calves. c Axial SE T1-weighted fat-saturated sequence through the left calf
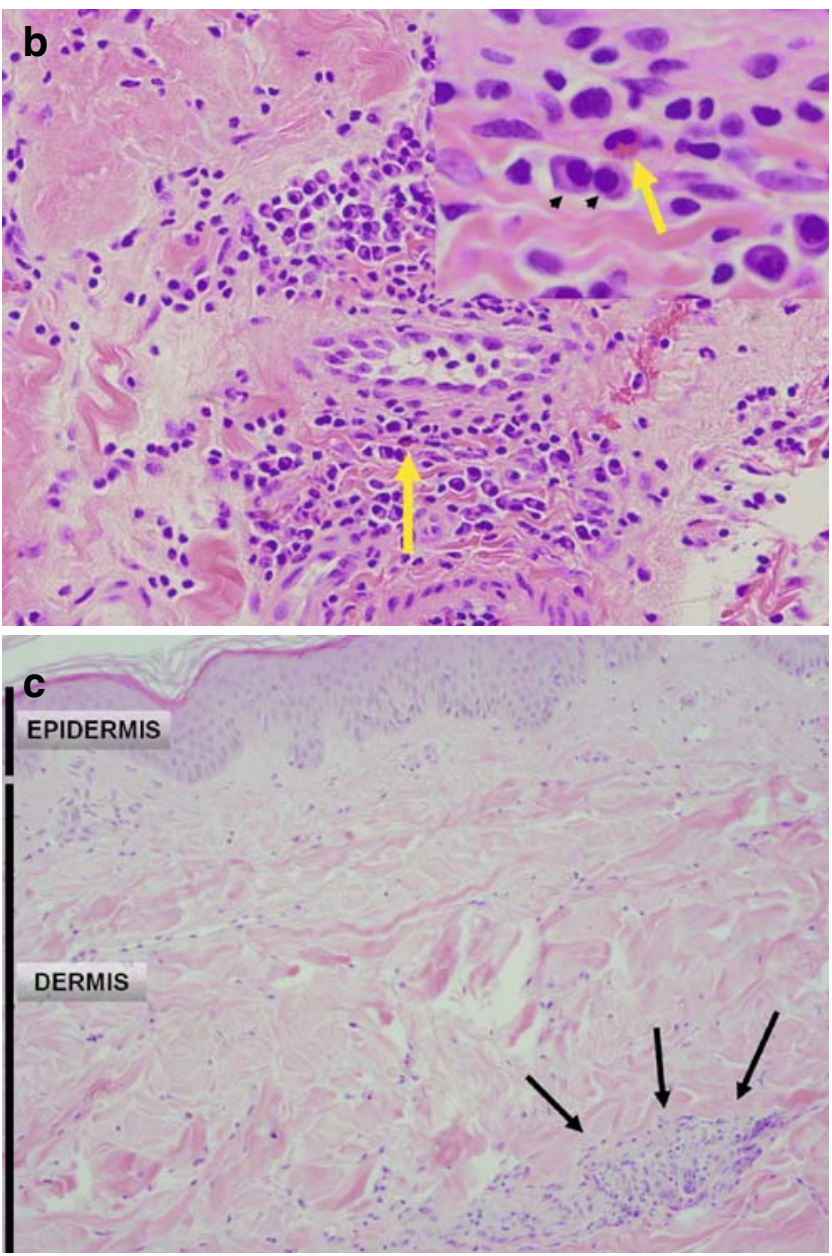

Fig. 3 a Histopathology image of the deep fascia $(H \& E, \times 100)$. b Histopathology image of the fascia $(\mathrm{H} \& \mathrm{E}, \times 200)$ with inset view $(H \& E, \times 400)$. c Histopathology image of the dermis 